ARTICLE OPEN

Check for updates

\title{
Determination of the dynamic cellular transcriptional profiles during kidney development from birth to maturity in rats by single-cell RNA sequencing
}

\author{
Fangrui Ding $\mathbb{I D}^{1,2,3 凶}$, Xiuying Tian $\mathbb{D}^{1,2,3}$, Jiali Mo $\mathbb{D}^{4}$, Botao Wang $\mathbb{D}^{4}$ and Jun Zheng $\mathbb{D}^{1,2,3 凶}$
}

(c) The Author(s) 2021

Recent single-cell RNA sequencing (scRNA-seq) analyses have offered much insight into the gene expression profiles in earlystage kidney development. However, comprehensive gene expression profiles from mid- and late-stage kidney development are lacking. In the present study, by using the scRNA-seq technique, we analyzed 54,704 rat kidney cells from just after birth to adulthood (six time points: postnatal days $0,2,5,10,20$, and 56) including the mid and late stages of kidney development. Twenty-five original clusters and 13 different cell types were identified during these stages. Gene expression in these 13 cell types was mapped, and single cell atlas of the rat kidney from birth to maturity (http://youngbearlab.com) was built to enable users to search for a gene of interest and to evaluate its expression in different cells. The variation trend of six major types of kidney cells-intercalated cells of the collecting duct (CD-ICs), principal cells of the collecting duct (CD-PCs), cells of the distal convoluted tubules (DCTs), cells of the loop of Henle (LOH), podocytes (PDs), and cells of the proximal tubules (PTs)-during six postnatal time points was demonstrated. The trajectory of rat kidney development and the order of induction of the six major types of kidney cells from just after birth to maturity were determined. In addition, features of the dynamically changing genes as well as transcription factors during postnatal rat kidney development were identified. The present study provides a resource for achieving a deep understanding of the molecular basis of and regulatory events in the mid and late stages of kidney development.

Cell Death Discovery (2021)7:162; https://doi.org/10.1038/s41420-021-00542-9

\section{INTRODUCTION}

Prematurity is the most common cause of hospitalization in neonatal intensive care units (NICUs) $[1,2]$. The major issue in preterm infants is the immaturity of organs and systems at birth and then abnormal developmental programs after birth when compared with full term infants, which strongly increase the risk of various long-term chronic diseases. This hypothesis is also called the developmental origins of health and disease ( $\mathrm{DOHaD})$, which proposes that deleterious events occurring in the earliest stages of human development affect the long term occurrence of diabetes, cardiovascular disease, asthma, neuropsychiatric disorders, chronic kidney disease (CKD), and so on [3-5]. In the field of kidney disease, Brenner et al. first proposed that a congenital decrease in nephron number could cause renal injury, which is similar to the $\mathrm{DOHaD}$ hypothesis $[6,7]$. This hypothesis provides a plausible explanation for the high prevalence of renal disease in low birth weight or preterm infants, who are expected to show a low number of nephrons [6, 7]. Currently, an increasing number of clinical studies have shown that prematurity is a risk factor for CKD [8-12]. However, the underlying molecular mechanism has not been elucidated.
Most preterm infants are born at 21 to 37 weeks of gestation corresponding to mid and late gestation [13, 14]. Before kidney development in preterm infants can be fully elucidated, the normal physiological gene expression patterns during this age should be determined. In recent years, single-cell RNA sequencing (scRNA-seq) has made a major contribution to elucidating developmental processes as well as mapping gene profiles during organ development, including kidney development [15-25]. Many studies have provided comprehensive and dynamic gene expression profiles during kidney development by using scRNA-seq $[16,18,20-22]$. However, most of these studies focused on embryonic kidney development and early fetal kidney development (corresponding to less than 20 gestational weeks). Few studies have focused on the mid and late stages of kidney development. Because it is difficult to acquire kidney samples during 21-37 gestational weeks in humans, murine can be used as a good model for elucidating the development molecular mechanism of this organ during this age.

Kidney development between murine and humans is different. In humans, nephrogenesis (the formation of nephrons) starts at 4-5 weeks of gestation and is completed in utero at gestational

\footnotetext{
${ }^{1}$ Department of Neonatology, Tianjin Central Hospital of Obstetrics and Gynecology, Tianjin, China. ${ }^{2}$ Tianjin Key Laboratory of Human Development and Reproductive Regulation, Tianjin, China. ${ }^{3}$ Department of Neonatology, Nankai University Maternity Hospital, Tianjin, China. ${ }^{4}$ Graduate School, Tianjin Medical University, Tianjin, China. 凶email: youngbear@126.com; zhengjunpaper@126.com Edited by A. Rufini
}

Received: 2 December 2020 Revised: 27 April 2021 Accepted: 3 June 2021

Published online: 24 June 2021 
week 36 before full-term birth. In mice and rats, nephrogenesis begins on embryonic days 11 and 12, respectively, and is completed at $\sim 20$ days after birth. Therefore, the difference between humans and murine is that nephrogenesis in humans is completed in utero before birth, while the murine kidney is still immature at birth, and nephrogenesis has both intra- and extra-uterine developmental stages [26-29]. The extra-uterine developmental stage of the murine kidney corresponds to mid and late gestational kidney development in humans. Because of this characteristic of extra-uterine development in mice and rats, acquiring kidney samples for further study is very convenient during this stage, and instead of calculating the precise embryonic age, researchers can wait for murine birth. Thus, a postnatal murine model could be the best model for determining the mid and late gestational development of the kidney. In the present study, a scRNA-seq of 54,704 renal cells spanning postnatal day 0 -postnatal day 56 (corresponding to mid, late, and mature stages of kidney development) was performed. Distinct cell populations, as well as gene expression profiles, were identified, and developmental trajectories of the major kidney cell populations were determined in this study. In addition, due to the limited amount of published gene expression data from the mid to late stages of renal development in any species, the sequence dataset has also been presented as a database at http://youngbearlab.com; this database is being provided as a resource for individual researchers to perform their own sophisticated analyses of questions of interest.

\section{RESULTS \\ Clustering of cells during postnatal kidney development of rats}

In the present study, six-time points were chosen for mapping the gene expression of postnatal kidney development in rats: postnatal days $0,2,5,10,20$, and 56 , which correspond to kidney development stages in most human preterm infants according to pathological findings. During postnatal day 0-postnatal day 5, nephrogenesis was clearly detected. At this age, the comma- and $\mathrm{S}$-shaped bodies were detected in the outer renal cortex. During postnatal day 10-postnatal day 20, obvious nephrogenesis was not detected in the kidney, while glomeruli were still immature. After postnatal day 20, most of the glomeruli, as well as the tubules, were mature [28-32]. To identify the gene expression profiles during these times, we performed single-cell sequencing and analysis. Finally, 54,704 cells were obtained, and uniform manifold approximation and projection (UMAP) analysis of the combined datasets yielded 25 original clusters, and the median expression levels of each gene in each cluster are provided in Supplement Table 1. According to the differentially expressed genes (DEGs) in the 25 original clusters (Supplement Table 2) and annotation by known gene markers, 13 different cell types were identified overall (Fig. $1 \mathrm{~A}$ and Fig. S1).

\section{Cellular diversity of rat kidney development after birth identified by single-cell transcriptomic analysis}

To define the identity of each cluster, the expression patterns of previously identified cell-type-specific markers in each cell cluster were examined. As shown in Fig. 1, cluster 0 represented proximal tubules (PTs), as these cells highly expressed the specific PT genes SLC27A2 and CUBN $[33,34]$. Cluster 1 highly expressed UMOD and SLC12A1 and belonged to the loop of Henle (LOH) $[35,36]$. Cluster 2 represented distal convoluted tubules (DCTs), as they highly expressed SLC12A3 [37]. Cluster 3 highly expressed principal cells of the collecting duct (CD-PCs) marker genes, such as AQP2 and AQP3 $[38,39]$. In cluster 4 , the proliferating cell 1 (PC1) marker genes TOP2A and CENPF were highly expressed [18]. For the identification of intercalated cells of the collecting duct (CD-ICs), ATP6V1G3 and ATP6V0D2 were selected, and cluster 5 was identified [17]. Podocytes (PDs) highly express NPHS1 and NPHS2, and cluster 6 was identified $[40,41]$. Stromal cells (SCs) highly express COL3A1 and LGALS1, which were identified in cluster 7 [42, 43]. Endothelial cells (ECs) are represented by cluster 8 , which highly expressed KDR and PLVAP $[44,45]$. Cluster 9 highly expressed S100A8 and S100A9 [46]; thus, this cluster represents immune cells (ICs). Cluster 11 represented a small group of urothelial cells (UCs), as these cells specifically express UPK3A [47]. HBB, ALAS2, and AHSP were highly expressed in cluster 12 , namely, erythroblasts (ERs) [48, 49].

There are three clusters that were difficult to annotate: original clusters 1, 20, and 21 (Supplement Table 2 and Fig. S1). For clusters 1 and 20, highly DEGs were CRYAB, TSPAN8, SPP1, IGFBP2, and SLC31A2. After annotation by several kidney studies and kidney databases such as GenitoUrinary Development Molecular Anatomy Project (GUDMAP) (This database contains a molecular atlas of gene expression for the developing organs of the GenitoUrinary tract and addresses at https:// www.gudmap.org/) and the Human Protein Atlas (HPA) (this database contains the distribution of the proteins across all major tissues and organs in the human body and expression of protein-coding genes in a single human cell type and addresses at https://www.proteinatlas.org/), we found that these genes are not limited to one known cell type [16-19, 22, 23]. They have been found to be expressed in tubules (LOH, PTs, DCTs, and CD-PCs). Then, we performed subclustering of these two clusters, as shown in Supplement Table 3. These cells were clustered into nine subclusters. According to studies to identify gene profiles of the separate kidney zones by Andrew Ransick et al. and Jae Wook Lee $[19,50]$, the top DEGs (TSPAN8, ID1, ID3, and JAG1) in subcluster 0 and subcluster 1 were highly expressed in short descending limbs of LOH. The top DEGs (CLDN10, BSND, and CLCNKA) in subcluster 2 were highly expressed in thin ascending limbs of LOH. The top DEGs (EGR1, FOS, and EHF) in subcluster 3 and subcluster 6 were highly expressed in thin descending limbs of LOH. Additionally, the top DEGs were also assessed with Kidney Cell Explorer (This explorer is a database of kidney single-cell database) [19]. As a result, most of these genes were highly expressed in the LOH cell population (Fig. S2). Thus, original clusters 1 and 20 were annotated as the LOH.

It was also difficult to annotate original cluster 21 (present cluster 10). We examined the expression pattern of the top DEGs such as HAS2 and WNT4 in all cells and viewed the results in UMAP. Most of these genes were expressed in proliferating cells (original cluster 11, present cluster 4) (Fig. S3A-D). In addition, based on cluster hierarchy (Build Cluster Tree function) (Fig. S3E), these two clusters originated from the same hierarchy. Then, this cluster was a highly likely proliferating cell. However, the marker genes TOP2A and CENPF highly expressed in cluster 4 were not found in this cluster; therefore, this cluster was annotated as proliferating cell 2 (PC2).

After annotation all clusters, we have also built a searchable database including a single-cell atlas of the rat kidney from birth to maturity (http://youngbearlab.com). Users can search for a gene of interest and evaluate its expression in different cells.

\section{Distinct phases of kidney development after birth}

For analysis of the dynamic changes in the cell population at different times, all the cells from six separate time points are shown in the UMAP graph (Fig. 2A). Total six major kidney cell populations, including CD-IC, CD-PC, DCT, LOH, PD, and PT have been normalized to $100 \%$ at the same time point and then each cell type proportion has been recalculated and presented in Fig. $2 \mathrm{~B}$. The changing trends of each cell type during the time were combined and presented in Fig. $2 \mathrm{C}$. As shown in Fig. 2B, $C$, the numbers of PDs, CD-PCs, and DCTs decreased during the postnatal period, while that of CD-ICs increased over time. The general trend of the percentage of the $\mathrm{LOH}$ was to decrease first and then to increase. The percentage of PT gradually increased from postnatal day 0 to postnatal day 10 . After postnatal day 10 , it decreased. 
A

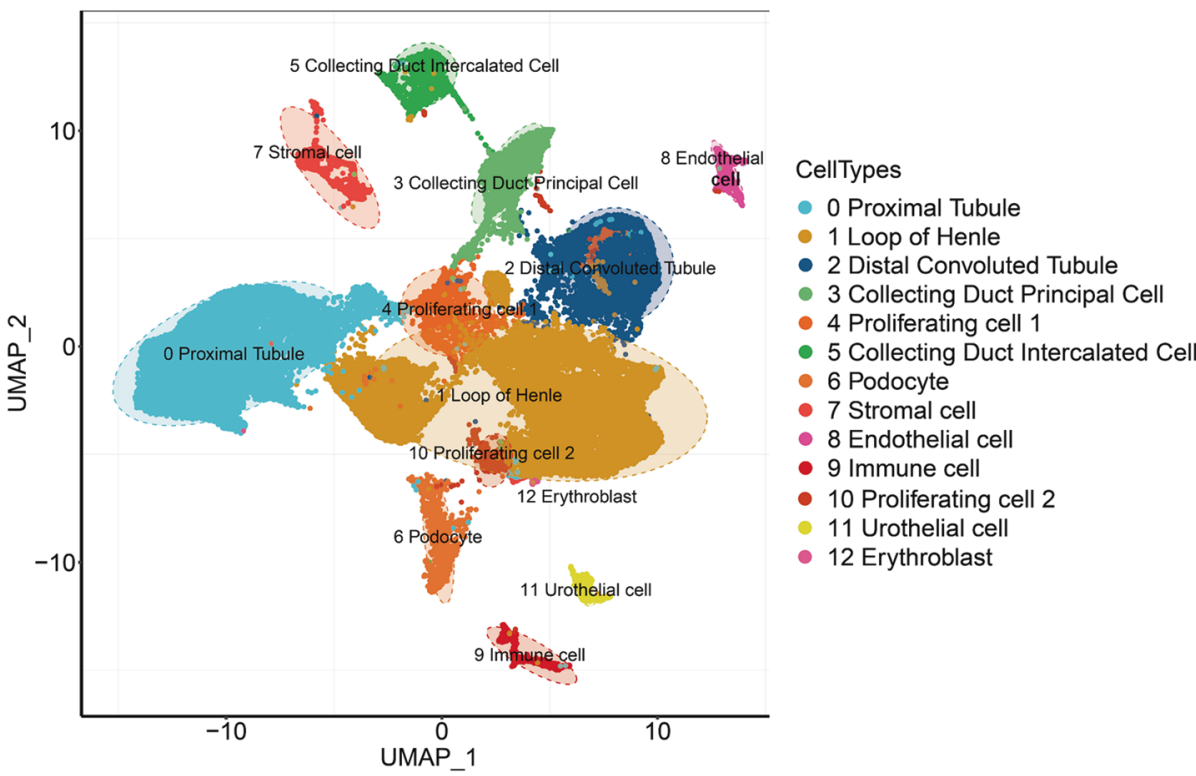

B

C $\begin{array}{llllllllllllll}0 & 1 & 2 & 3 & 4 & 5 & 6 & 7 & 8 & 9 & 10 & 11 & 12\end{array}$
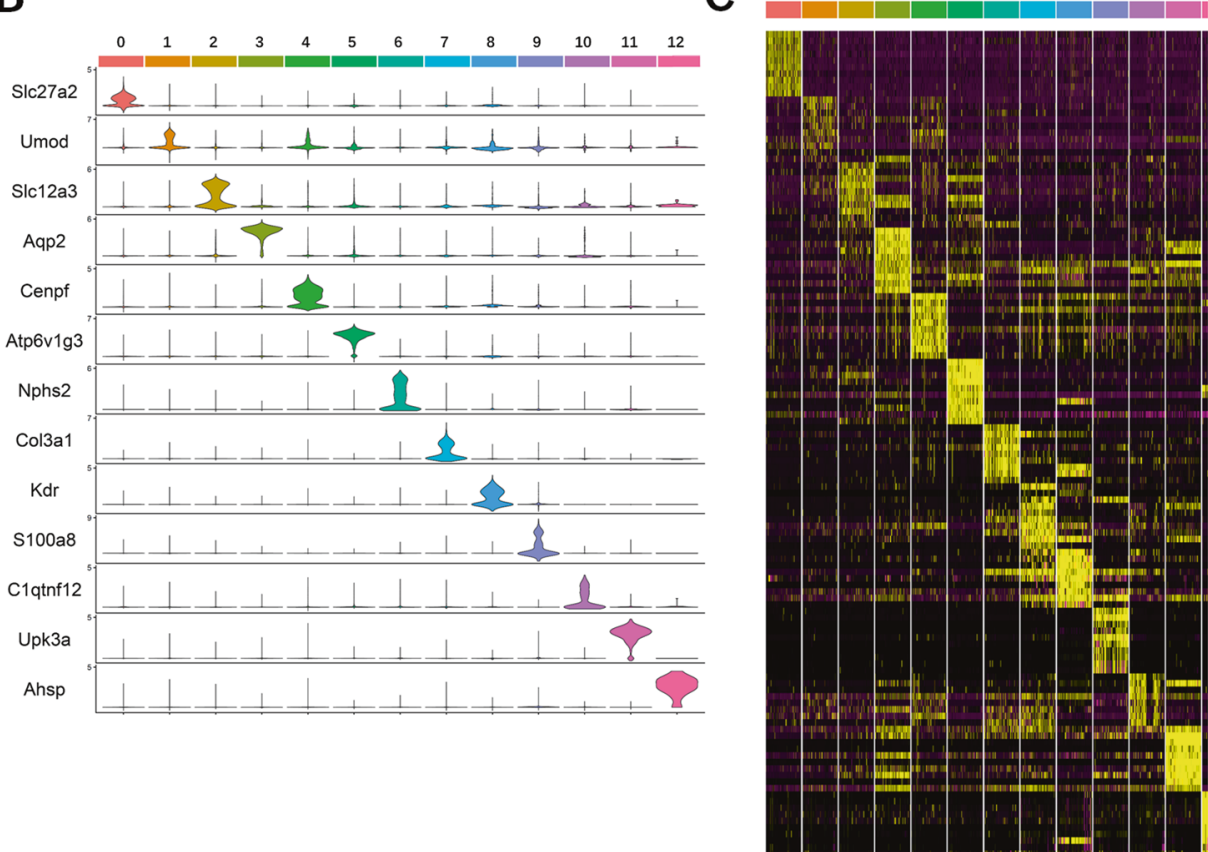

Cell types during the development of the postnatal rat kidney delineated by single-cell transcriptomic analysis. A Postnatal rat kidneys were collected at days $0,2,5,10,20$, and 56 after birth, and after preparation, single-cell suspension sequencing and analysis were performed. Thirteen distinct cell clusters revealed by unsupervised clustering and shown in a two-dimensional UMAP map. A total of 54,704 cells and 25 original clusters were identified. After the analysis of differentially expressed genes in the 25 original clusters with known gene markers, 13 different cell types were identified and annotated, as shown on the right side of UMAP results. B Violin plots showing the expression level of representative marker genes across the 13 clusters. The vertical axis shows the gene expression value. Each color represents one cluster as indicated on the right side in $\mathbf{C}$. The annotations of each color are presented on the right side of $\mathbf{C}$. $\mathbf{C}$ Heatmap of the gene expression patterns of the top ten cluster-specific genes in 13 clusters. Different clusters are identified by different colors, as indicated at the top of the heatmap and annotated on the right side of the heatmap. The color bar ranging from yellow to red-purple reflects the relative expression levels from high to low.

\section{Pseudotemporal ordering of major kidney cells during postnatal kidney development}

Previously, single-cell sequence analyses have revealed that the early stage of kidney development corresponds to the early gestational weeks $[16,18-20,22]$. To elucidate gene expression dynamics during postnatal to mature kidney development, we performed pseudotemporal ordering of the major kidney cells (PD, $\mathrm{PT}, \mathrm{LOH}, \mathrm{DCT}, \mathrm{CD}-\mathrm{PC}$, and $\mathrm{CD}-\mathrm{IC}$ ) in the present study. As shown in Fig. $3 A, B$, the initiation period was not limited to one or two cell populations because of the period of time of samples we chose. This period contained PD, LOH, DCT, and CD-PC. There are two major paths during postnatal rat kidney development. One 

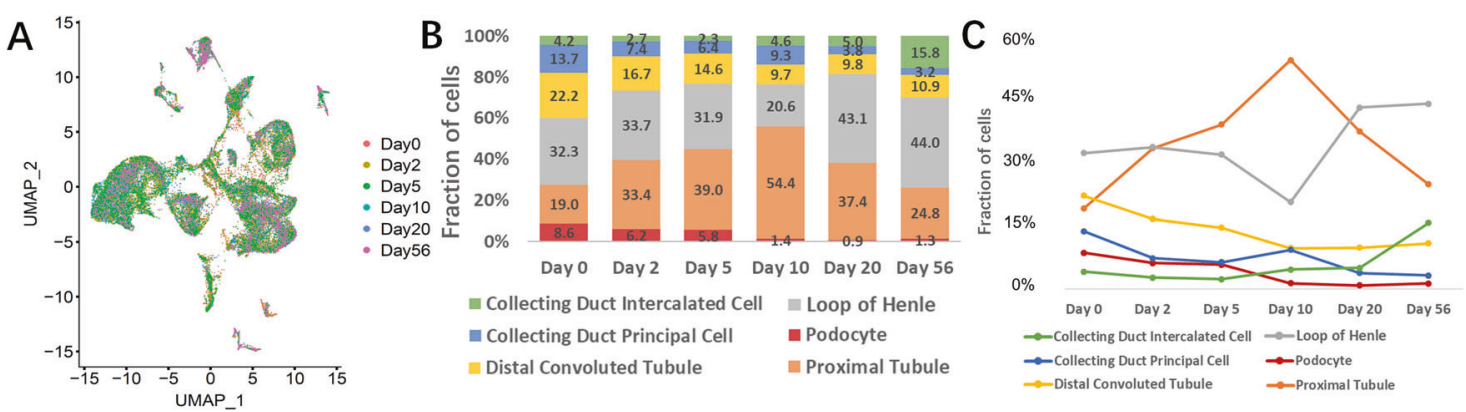

Fig. 2 Dynamic changes in major kidney cell types over time. A UMAP plots of six different developmental stages of rat kidney after birth. B The six major kidney cell types, namely, collecting duct intercalated cell, collecting duct principal cell, distal convoluted tubule, loop of Henle, podocyte, and proximal tubule were normalized to $100 \%$ at the same time point. The proportions of each cell type at each point are stacked, and dynamic changes are presented over development time. C The change trends of the proportion of each major kidney cell type during different developmental stages are indicated with broken lines. Podocyte, collecting duct principal cells, and distal convoluted tubules showed decreases during the postnatal period while collecting duct principal cells show an increase over time. Loop of Henle showed a decrease from postnatal days 0 to 10 and then an increase, while proximal tubules present the opposite pattern.

involves CDs, LOHs, and DCTs. Another involves PTs. As shown in Fig. $3 C$, the split trajectories of each cell type showed that PD was the first cell type presented and fated to mature, followed by $\mathrm{LOH}$ and CD-PC, which were ordered by DCT and CD-IC. Finally, PT was induced to mature during kidney development. Previous study has shown that nephron progenitor cells are induced to two major distinct trajectories: PDs and proximal/distal tubule fates [21, 22]. In those studies, PDs are induced from progenitor cells earlier than tubules [21, 22], which is same with our founding. In detail, the accurate order was determined in this study when combined with previous studies describing the state from progenitor cells to maturity (Fig. 3D).

\section{Features of dynamically changing genes during postnatal rat kidney development}

To further map the gene expression dynamics and to find potential key genes of signaling pathways during postnatal kidney development, we analyzed DEGs during postnatal kidney development along the pseudotime. First, the top five DEGs in pseudotime were identified, and $3 / 5$ were genes that encode ribosomal proteins and related proteins (Fig. 4A, B). The percentage was unexpectedly high. Then, the top 50 DEGs in pseudotime were identified (Supplement Table 4). These genes accounted for 30\% (15/50) of the DEGs. As shown in Fig. 4B, C, at the start of postnatal kidney development in rats, these genes were highly expressed and gradually decreased during pseudotime. Ribosomal proteins are known to be involved in protein synthesis [51-53]. The dynamic changes in these genes suggested that high levels of proteins were produced during the mid and late stages of kidney development. In addition to ribosomal proteins, the top 200 DEGs of each major kidney cell type (CD-IC, CD-PC, DCT, LOH, PD, and PT) (Supplement Table 5) except ribosomal proteins from pseudotemporal ordering analysis were chosen by using GO analysis. As shown in Fig. S4, in the GO analysis, most of the GO terms were involved in translational and posttranslational processes including proteasome complex, methylosome, and chaperone complex in the cellular component module (Fig. S4) and translation factor activity, RNA binding, unfolded protein binding, and ubiquitin protein ligase binding in the molecular function module (Fig. S4 and Supplement Table 6), which suggested that different cell types complete their fate and then initiate a large number of functional unit synthesis of each lineage during mid and late stages of kidney development [16, 21]. In addition, DNA-binding transcriptional factors (TFs) can play roles in translational regulation during development. Figure 4D lists the top five TFs among the top 200 DEGs over pseudotime. They include TCF21, FOXD1, WT1, MAFB, and JUN. Moreover, the TFs enriched in six major kidney cell types are also listed in Fig. 4E.
Cell-type-specific expression of CKD-related genes. Prematurity is a risk factor for CKD [8-12]. Though there are many possible causes of CKD, the major cause is glomerular diseases [54]. The known glomerular disease genes were collected from the OMIM database, and the expression features of these genes were examined during these stages. There are three major cell types in the glomerulus: PDs, ECs, and mesangial cells. In the present study, intraglomerular ECs and mesangial cells were not separated from total ECs and stroma cells. Hence, these genes were examined in PDs, ECs, and stroma cells. As shown in Figs. 5, S5, most of these genes were highly expressed in these three types of cells, among which PDs accounted for the largest percentage.

\section{DISCUSSION}

In humans, normal nephrogenesis is completed in utero in 36 weeks, indicating that most preterm infants experience a potential abnormal extra-utero developmental stage [26]. The gestational weeks of most preterm infants range from 21 to 37 weeks $[13,14]$, which corresponds to the mid and late developmental stages of the kidney. Many studies have focused on embryonic kidney development, early fetal kidney development, or mapping the gene expression profile of the mature kidney. In a study by Lindstrom et al., researchers clearly identified a time-dependent process of cell fate in early nephrogenesis [21]. In a study by Wang et al., early fetal kidney development was explored [16]. In addition, Park et al. mapped mature kidney gene expression patterns [17]. However, few studies have explored the mid and late stages of kidney development to maturity. To our knowledge, the present study may be the first to reveal the dynamic physiological gene expression profiles of the rat kidney from birth to maturity based on scRNA-seq analysis. In addition, the sequence data from the present study will serve as a resource for generating an atlas of gene expression during these stages. Considering the limited knowledge about all aspects of kidney development described herein, other researchers will be most welcome to use our data to reveal more details during these stages. To ensure that our sequence data are available to every potential user, we have generated a web database to facilitate access to our data and analysis of these data by the academic and clinical research communities. Users can search for the gene of interest and evaluate the expression in different cells. Additionally, this resource was produced in rat; in the future, it is expected that human data during the mid- and late developmental stages will be made available and that comparisons of rat and human data will be performed to explore the differences between these two species [55]. 

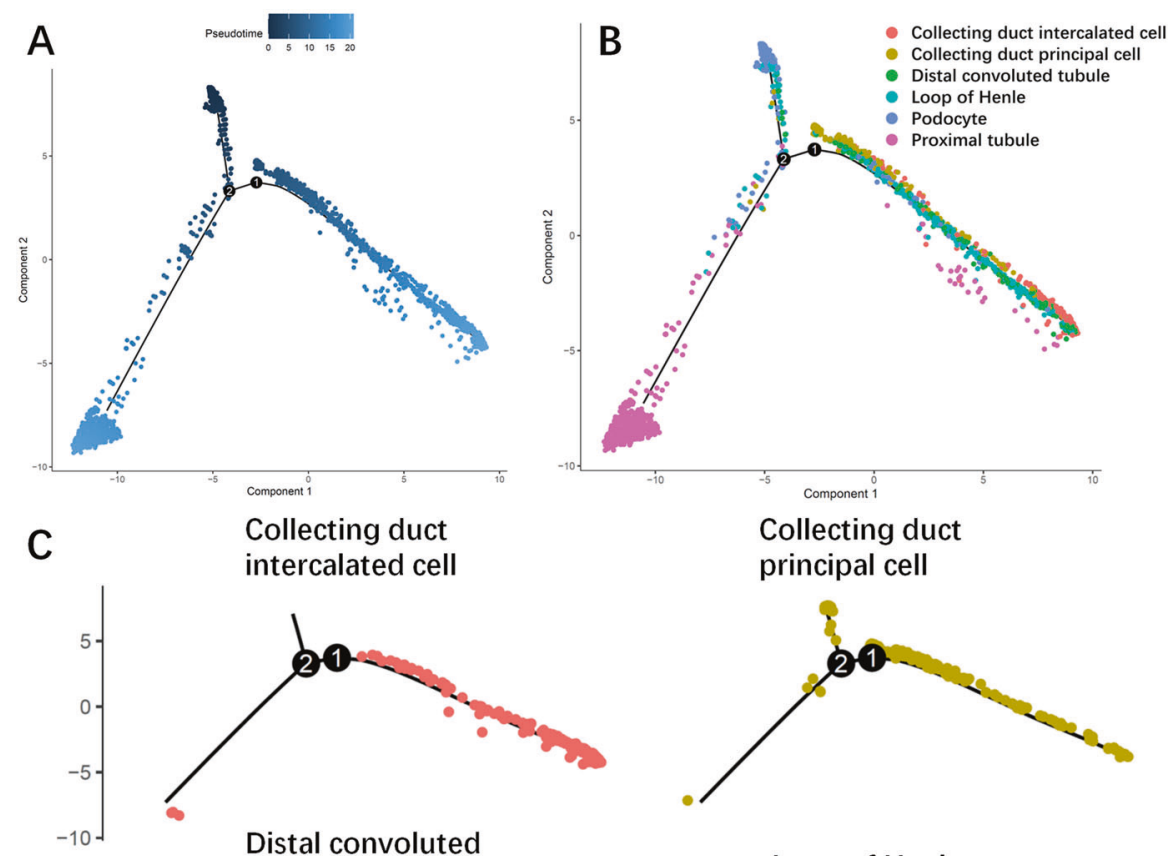

Collecting duct principal cell
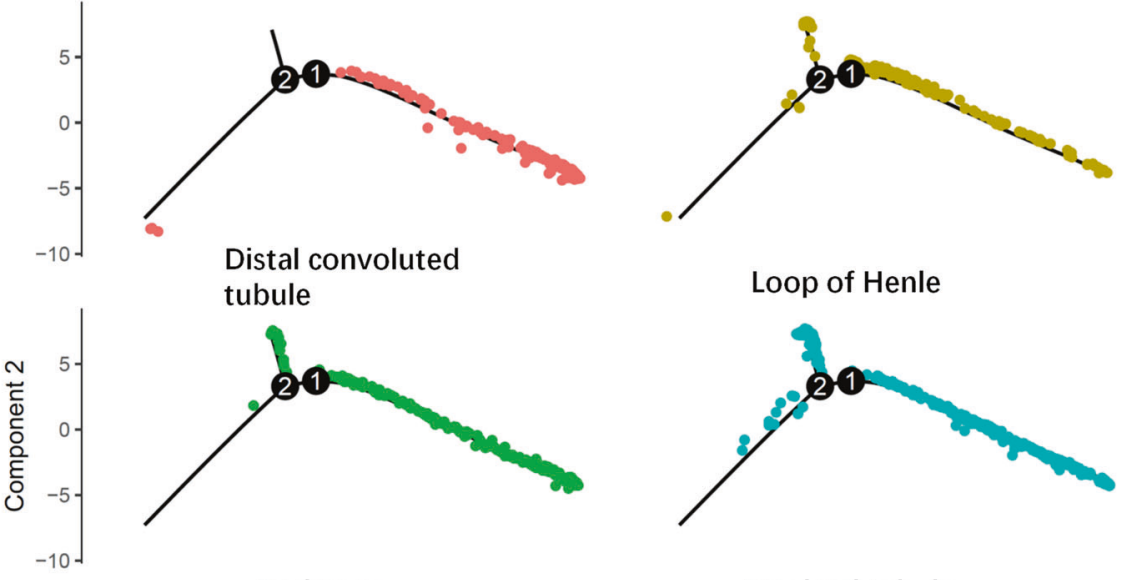

Distal convoluted

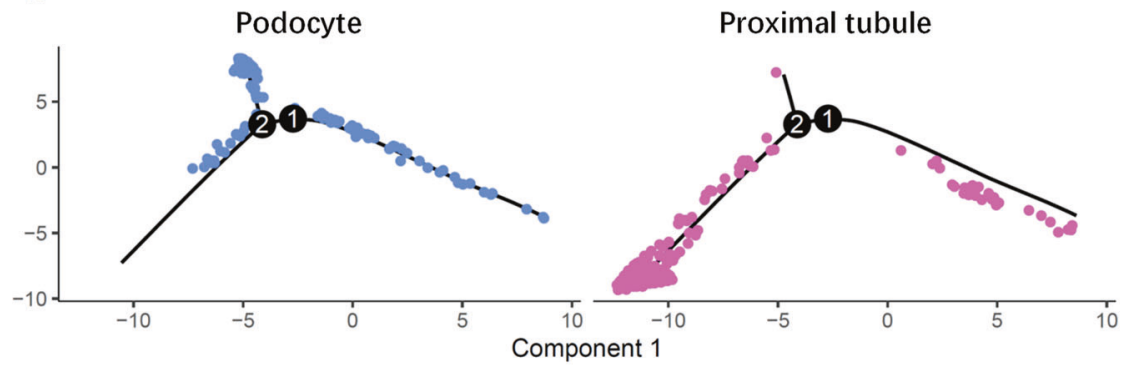

Loop of Henle

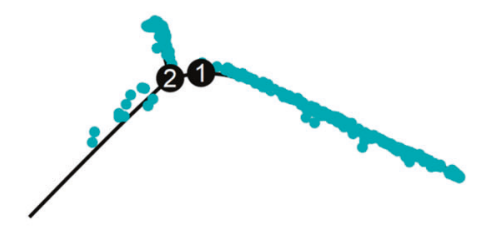

D

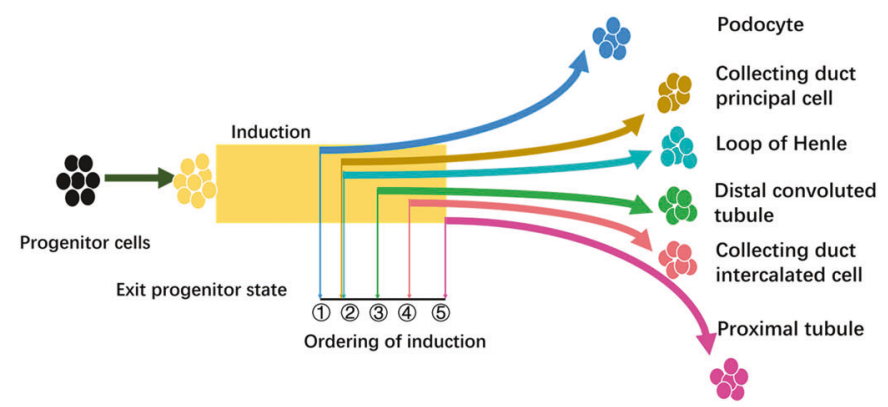

Fig. 3 Distinct phases of major kidney cell clusters during the development of postnatal rat kidney. A, B Pseudotime trajectory of the major kidney cells (collecting duct intercalated cells, collecting duct principal cells, distal convoluted tubules, loop of Henle, podocytes, and proximal tubules). Cells are colored based on the predicted pseudotime. The deeper the color, the earlier it is (A). Cell type transition during the development of rat kidney after birth according to pseudotime path (B). C Split monocle pseudotime plot revealed detailed development of the major kidney cell clusters. D Overview of different cell cluster fates along cumulative time as indicated by pseudotime. Numbers $1-5$ present the ordering of cell type induction based on pseudotime. Podocytes were the first cell type to be induced from the progenitors and to reach their mature fate, followed by the loop of Henle and collecting duct principal cells, and then distal convoluted tubules and collecting duct intercalated cells, with proximal tubules being the final mature cell type observed kidney development.

During the mid and late stages of kidney development, the development of the $\mathrm{LOH}$ is one of the major developmental processes $[18,19,56,57]$. In the present study, the LOH exhibited the largest number of cells $(19,651 / 54,704=35.9 \%)$, which confirmed the possible key role during mid and late kidney development. Thus, negative factors occurring during the mid and late stages of kidney development may also cause an adverse effect on the $\mathrm{LOH}$. The LOH plays a role in successful radiation, and short 


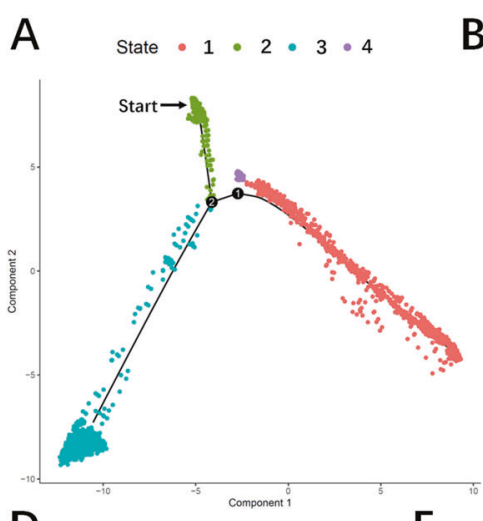

D

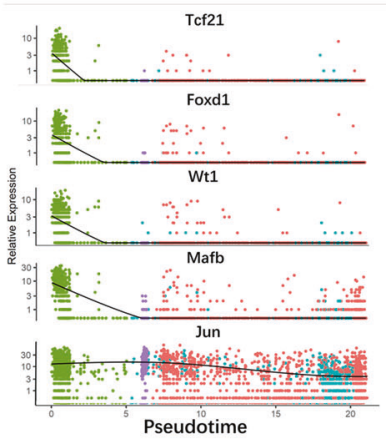

B

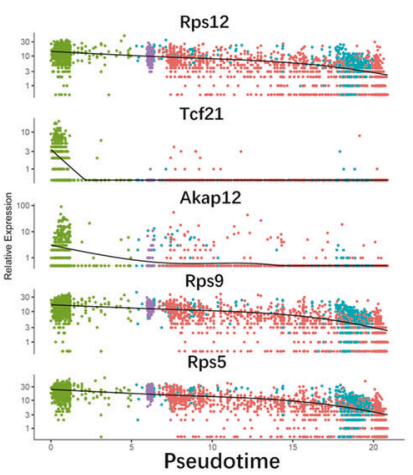

E

Collecting duct intercalated cell TFs

Foxi1 Dmrt2 Pbx3 Gata3 Id3 KIf4 Tox3 Esrrg Id1 Id2 Id4 Elf3 Gata2 Cebpd Ddit3 Hmx2
C

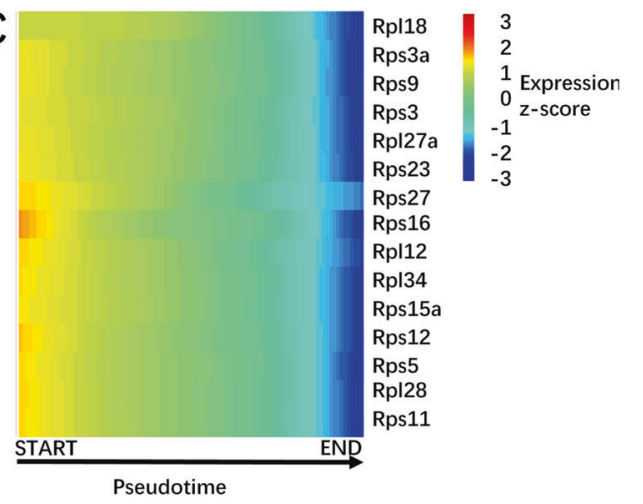

Collecting duct principal cell TFs

Id3 Id1 Fos Egr1 Gata3 Junb Atf3 Fosb Zfp36I1

Nfe2l2 Id4 Jun Elf3 KIf5 Jund Id2 Gata2 Plagl1 Tox3

En2 Cebpb Heyl Hoxd9 Hmgb2 Nr4a1 Nfat5 Scx

Hes1 Zfp36I2 Maff Est2 Ehf Tgif1 Csrnp1 Egr2 Egr4

Distal convoluted Loop of Henle TFs tubule TFs

Emx1 Tox3

Hoxd9 Gata3

Zfp503 Vdr

Tfap2b Mecom

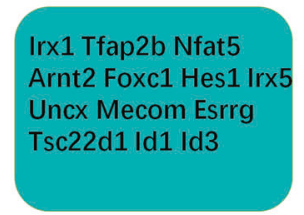

Podocyte TFs

Mafb Foxd1 Wt1 Tcf21

Carhsp1 Foxc2 Peg3 Foxc1

Foxs1 Tsc22d3 Jun Jund

Fos Fosb Osr2 Junb Meis2

Zfp36I2 Egr1 Tsc22d1
Proximal tubule TFs

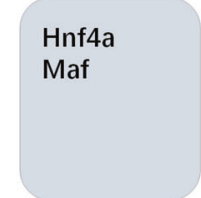

Fig. 4 Features of dynamically changing genes over temporal trajectories. A Cell state transition according to the pseudotime path. There are four major states over pseudotime. B Expression patterns of the top five DEGs over pseudotime. C Heatmap of ribosomal protein genes in the top 50 DEGs of the pseudotime path are significantly changed in the pseudotime path. All of these genes were highly expressed at the earlier stage and decreased during time. D Top five TFs in the top 200 DEGs over the pseudotime are listed and the expression patterns of these TFs over pseudotime were mapped. E TFs enriched in the six major kidney cell types are listed. The genes highlighted in red in each box were top five TFs among the top 200 DEGs over the pseudotime. All of the top five TFs were enriched in podocytes indicating the important role of podocytes during these development stages. DEGs differentially expressed genes, TFs transcriptional factors.

loop nephrons have been associated with smaller glomeruli [56]. Previous studies have shown that preterm individuals have a reduced nephron number and increased number of abnormal glomeruli $[6,7]$. Though little is known in preterm infants regarding whether the $\mathrm{LOH}$ affects abnormal glomeruli, we still considered that $\mathrm{LOH}$ development plays an important role in preterm kidney developmental processes. In addition, experts on the $\mathrm{LOH}$ are encouraged to access our database for further research.

In addition to the gene expression profile presented in this study, the time development process of different kidney cell types was shown. Furthermore, the DEGs during pseudotime were examined. In the top 50 DEGs during pseudotime, genes that encode ribosomal proteins accounted for a large percent. Ribosomes are organelles for protein synthesis [51-53]. A large number of ribosomal protein-related genes have been highlighted, which suggests that high protein levels are produced at the early stage of postnatal development compared with the mature stage. In addition to ribosomal proteins, GO analyses except for ribosomal proteins from pseudotemporal ordering analysis were also performed. Most of the GO terms were involved in translational and posttranslational processes (Fig. S4 and Supplement Table 6). These results indicated the importance of protein synthesis and protein modification and correspond to the formation of a larger number of functional units and organ size enlargement during the mid and late stages of development $[58,59]$. RPS12 is one of ribosomal related protein, which is also the top DEG during pseudotime in the present study. Though the role of this gene in kidney development has not been examined, a study in Drosophila strongly suggested that RPS12 is a determinant for organ growth and size in development [60]. In preterm infants, the functional unit nephrons were found in reduced numbers $[6,7]$ and further experimental studies should examine whether ribosomal proteins have an effect on nephron number and kidney size in preterm infants.

The TFs among the top 200 DEGs during pseudotime were listed. FOXD1, one of top five TFs among the top 200 DEGs over pseudotime, has already been shown to be a specific marker in stromal progenitor cells and is essential for normal glomerular development [61, 62]. In addition, MAFB is required for the differentiation of glomerular visceral epithelial cells during kidney development [63]. TCF21, another top five TF, was the first tissuerestricted basic helix-loop-helix protein identified in the developing kidney, and it plays a role in the regulation of morphogenetic events during kidney development [64]. WT1 has been confirmed to play an important role not only in kidney development but also in the pathogenesis of kidney diseases [65]. In addition, JUN has been reported to be involved in cell proliferation during kidney development [66]. All of the top five TFs among the top 200 DEGs over pseudotime play key roles in kidney development, which indicated that our study has a potential role in identifying or predicting important molecular factors involved in kidney development. Thus, the remaining differentially expressed TFs presented in our study could be the candidate key TF during the mid and late stages of kidney development. We have already listed these TFs in six major kidney cell types. These TFs are resources included in our database. Further experimental studies could also focus on these candidate TFs.

\section{MATERIALS AND METHODS}

\section{Animals}

Animal experiments were approved by the Tianjin Central Hospital of Gynecology and Obstetrics Animal Ethics Committee. All animal 


\section{$\begin{array}{lllllllllllllllll}0 & 1 & 2 & 3 & 4 & 5 & 6 & 7 & 8 & 9 & 10 & 11 & 12\end{array}$}

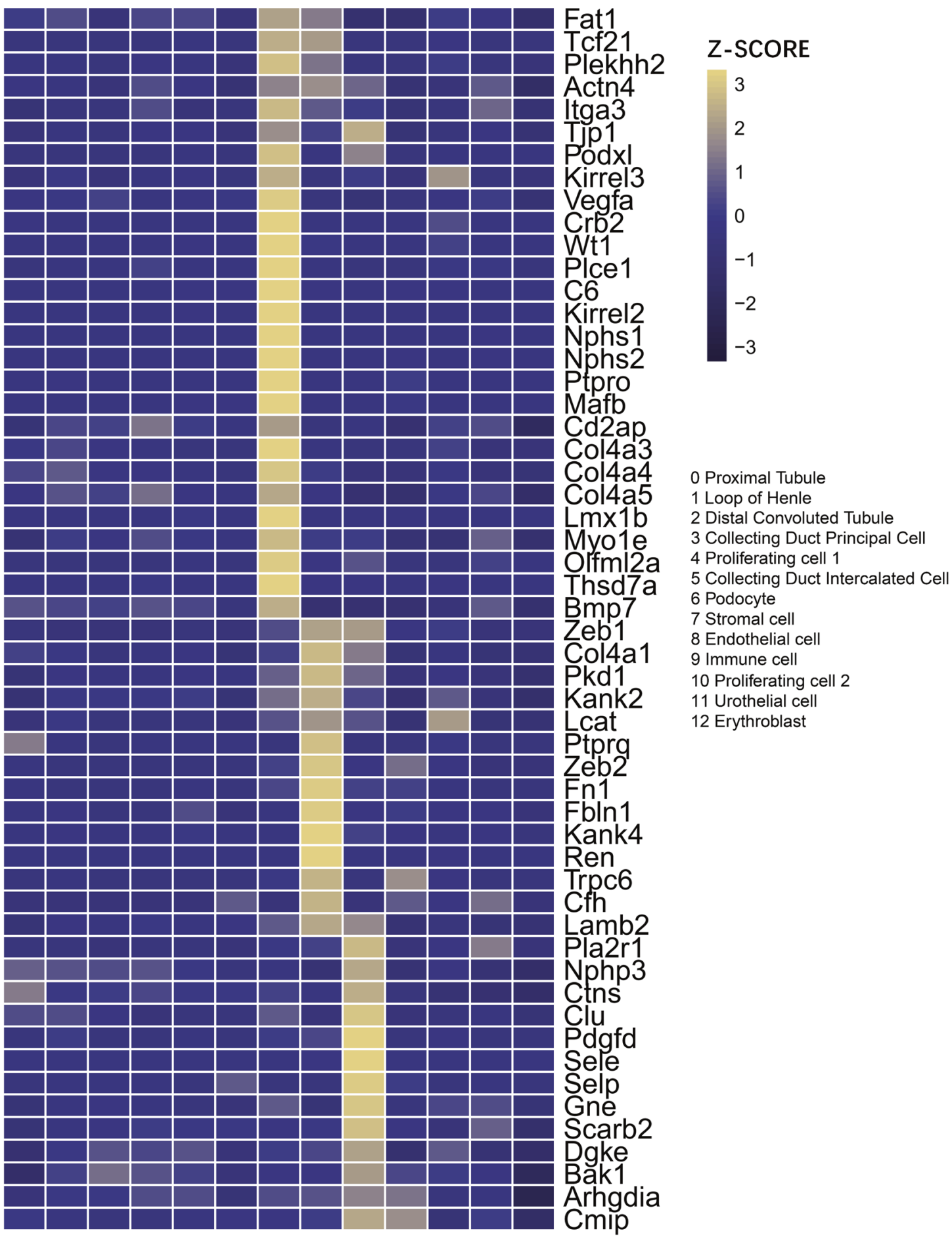

Fig. 5 Expression features of known glomerular diseases genes. Clustered expression of known glomerular disease genes collected from OMIM databases and genes showing high expression in podocytes, endothelial cells, and stroma cells are listed. Color keys indicate relative expression level and based on the $z$-score distribution. In the heatmap, each row represents one gene and each column is a single cell type. The full list of known glomerular disease genes and cell type is shown in Fig. S5.

experimental protocols conformed to the Institutional Animal Care Guidelines. Pregnant Sprague-Dawley rats were purchased from Charles River Laboratories (Beijing, China). At each time point, three rats (pups) at each time point were anesthetized by $\mathrm{CO}_{2}$ inhalation, and then their kidneys were pooled as one sequencing sample. The sex of the pups was not determined before weaning (P0, P2, P5, P10, and P20). P56 male rats were used as a mature kidney control.

\section{Single cell isolation}

For each sample, intact kidneys (from rats at postnatal days $0,2,5,10$, and 20) or $\sim 3.0 \mathrm{~g}$ of kidney tissue containing both the cortex and medulla (rats at postnatal day 56) was minced into millimeter-sized pieces in DMEM/F12 (Gibco A4192002) containing 10\% FBS (Gibco 10099141C). Then, the cells were digested in DMEM/F12 containing $1 \mathrm{mg} / \mathrm{ml}$ collagen type II (Sigma C6885) and $100 \mathrm{U} / \mathrm{ml}$ DNase I (Roche 4716728001) for $15-25 \mathrm{~min}$ at $37^{\circ} \mathrm{C}$ 
with agitation. The samples were pipetted up and down every $5 \mathrm{~min}$, and $20 \mu \mathrm{l}$ samples were used to assess the digestion. After digestion, the fragments were filtered through a $40-\mathrm{mm}$ nylon cell strainer (BD Falcon). The cell suspension was then centrifuged at $800 \mathrm{rpm}$ for $5 \mathrm{~min}$. After the removal of the supernatant, the cell pellets were washed three times with DPBS, resuspended in $5 \mathrm{ml}$ of red blood cell lysis buffer (Miltenyi 130-094183), and incubated for $2 \mathrm{~min}$ to remove red blood cells. Finally, the cells were resuspended by adding an appropriate volume of DPBS.

\section{Generation and sequencing of the 10x genomics RNA library} According to the manufacturer's instructions for the 10X Genomics system, the cell suspension was input into a 10X Genomics Chromium device to capture 8000-12,000 cells. Then, barcoding and CDNA synthesis were performed, and a unique molecular identifier (UMI) was added to identify PCR duplicates. Thereafter, the samples were incubated with appropriate enzymes to produce full-length CDNA, and PCR amplification was conducted to generate sufficient quantities of material for library construction. The CDNA libraries were constructed and sequenced by Illumina NovaSeq.

\section{Data processing and analyses}

After sequencing data were generated, quality control was first performed. For removal of potential doublets and low-quality cells, cells with $<200$ or $>3000$ unique expressed genes (as they are potential cell duplicates) were excluded, and genes expressed in ten or more cells were used for further analysis. Cells were also discarded if their mitochondrial gene percentages were over $70 \%$. Finally, 25,400 genes across 54,704 cells were generated for further analysis.

After the application of quality control filters, the filtered gene-barcode matrix of all samples was integrated with Harmony to remove batch effects [67]. Then, the Seurat R package v3.1 was used for dimensionality reduction analysis. The normalized data function was used to divide the total number of reads in the cell, multiplying by a scale factor of 10,000 and taking the log2-transformed values. The top 2000 genes showing the greatest variability were then identified using the "vst" method under the Seurat Find Variable Features function, and these top 2000 genes were used to recover the same clusters. Then, we performed principal component analysis (PCA) using the variable genes as input, and 30 statistically significant principal components were applied for UMAP. The cells were clustered using the Find Clusters function, and clusters were generated by differential expression analysis (DEA). The clusters were viewed in UMAP clustering. The unsupervised clustering was performed at a resolution level of 0.5 and resulted in 25 clusters (Supplement Tables 1, 2 and Fig. S1). After identification by well-known cell type markers, the same cell types were combined, resulting in 13 clusters. Each cell type contains hundreds to thousands of cells indicating the number of biological repeats.

In the pseudotime trajectory analysis, the Monocle2 (version 2.10.0) algorithm was used to identify cell transitions. The major kidney cell types, namely, PDs, PTs, DCTs, LOH, CD-PCs, and CD-ICs, were used for pseudotime trajectory analysis. Additionally, significantly changed genes over the pseudotime were identified. Gene Ontology (GO) analysis was performed with clusterProfiler, which supports statistical analysis and visualization of functional profiles for genes and gene clusters.

\section{DATA AVAILABILITY}

The datasets generated and/or analyzed during the current study are available in the Sequence Read Archive: PRJNA649702.

\section{REFERENCES}

1. Vogel JP, Chawanpaiboon S, Moller AB, Watananirun K, Bonet M, Lumbiganon P. The global epidemiology of preterm birth. Best Pract Res Clin Obstet Gynaecol. 2018;52:3-12.

2. Frey HA, Klebanoff MA. The epidemiology, etiology, and costs of preterm birth. Semin Fetal Neonatal Med. 2016;21:68-73.

3. Hanson MA, Gluckman PD. Developmental origins of health and disease-global public health implications. Best Pract Res Clin Obstet Gynaecol. 2015;29:24-31.

4. Feng A, Wang L, Chen X, Liu X, Li L, Wang B, et al. Developmental origins of health and disease $(\mathrm{DOHaD})$ : implications for health and nutritional issues among rural children in China. Biosci Trends. 2015;9:82-7.

5. Kajee N, Sobngwi E, Macnab A, Daar AS. The developmental origins of health and disease and sustainable development goals: mapping the way forward. J Dev Orig. Health Dis. 2017;9:1-5.
6. Mackenzie HS, Brenner BM. Fewer nephrons at birth: a missing link in the etiology of essential hypertension? Am J Kidney Dis. 1995;26:91-8.

7. Brenner BM, Chertow GM. Congenital oligonephropathy and the etiology of adult hypertension and progressive renal injury. Am J Kidney Dis. 1994;23:171-5.

8. Hallan S, Euser AM, Irgens LM, Finken MJJ, Holmen J, Dekker FW. Effect of intrauterine growth restriction on kidney function at young adult age: the Nord Trondelag Health (HUNT 2) Study. Am J Kidney Dis. 2008;51:10-20.

9. Keijzer-Veen MG, Schrevel M, Finken MJJ, Dekker FW, Nauta J, Hille ETM, et al. Microalbuminuria and lower glomerular filtration rate at young adult age in subjects born very premature and after intrauterine growth retardation. J Am Soc Nephrology. 2005;16:2762-8.

10. Vikse BE, Irgens LM, Leivestad T, Hallan S, Iversen BM. Low birth weight increases risk for end-stage renal disease. J Am Soc Nephrology. 2008;19:151-7.

11. Hodgin JB, Rasoulpour M, Markowitz GS, D'Agati VD. Very low birth weight is a risk factor for secondary focal segmental glomerulosclerosis. Clin J Am Soc Nephrology. 2009;4:71-6.

12. Ikezumi $Y$, Suzuki T, Karasawa T, Yamada T, Hasegawa $H$, Nishimura $H$, et al. Low birthweight and premature birth are risk factors for podocytopenia and focal segmental glomerulosclerosis. Am J Nephrol. 2013;38:149-57.

13. Stoll BJ, Hansen NI, Bell EF, Walsh MC, Carlo WA, Shankaran S, et al. Trends in care practices, morbidity, and mortality of extremely preterm neonates, 1993-2012. JAMA 2015;314:1039-51.

14. Patel RM, Kandefer S, Walsh MC, Bell EF, Carlo WA, Laptook AR, et al. Causes and timing of death in extremely premature infants from 2000 through 2011. N Engl J Med. 2015;372:331-40.

15. Tang F, Barbacioru $C$, Wang $Y$, Nordman E, Lee $C$, Xu N, et al. mRNA-Seq wholetranscriptome analysis of a single cell. Nat Methods. 2009;6:377-82.

16. Wang $P$, Chen $Y$, Yong J, Cui $Y$, Wang $R$, Wen $L$, et al. Dissecting the global dynamic molecular profiles of human fetal kidney development by single-cell RNA sequencing. Cell Rep. 2018;24:3554-67.

17. Park J, Shrestha R, Qiu C, Kondo A, Huang S, Werth $M$, et al. Single-cell transcriptomics of the mouse kidney reveals potential cellular targets of kidney disease. Science. 2018;360:758-63.

18. Lindström NO, Guo J, Kim AD, Tran T, Guo Q, De Sena Brandine G, et al. Conserved and divergent features of mesenchymal progenitor cell types within the cortical nephrogenic niche of the human and mouse kidney. J Am Soc Nephrology. 2018;29:806-24.

19. Ransick A, Lindström NO, Liu J, Zhu Q, Guo JJ, Alvarado GF, et al. Single-cell profiling reveals sex, lineage, and regional diversity in the mouse kidney. Dev Cell. 2019;51:399-413.

20. Adam M, Potter AS, Potter SS. Psychrophilic proteases dramatically reduce singlecell RNA-seq artifacts: a molecular atlas of kidney development. Development. 2017;144:3625-32.

21. Lindström NO, De Sena Brandine G, Tran T, Ransick A, Suh G, Guo J, et al. Progressive recruitment of mesenchymal progenitors reveals a time-dependent process of cell fate acquisition in mouse and human nephrogenesis. Dev Cell. 2018;45:651-60.

22. Menon R, Otto EA, Kokoruda A, Zhou J, Zhang Z, Yoon E, et al. Single-cell analysis of progenitor cell dynamics and lineage specification in the human fetal kidney. Development 2018;145:dev164038.

23. Fu J, Akat KM, Sun Z, Zhang W, Schlondorff D, Liu Z, et al. Single-cell RNA profiling of glomerular cells shows dynamic changes in experimental diabetic kidney disease. J Am Soc Nephrology. 2019;30:533-45.

24. Chen L, Lee JW, Chou CL, Nair AV, Battistone MA, Păunescu TG, et al. Transcriptomes of major renal collecting duct cell types in mouse identified by singlecell RNA-seq. Proc Natl Acad Sci USA. 2017;114:E9989-E98.

25. Lu Y, Ye Y, Yang Q, Shi S. Single-cell RNA-sequence analysis of mouse glomerular mesangial cells uncovers mesangial cell essential genes. Kidney Int. 2017:92:504-13.

26. Ryan $D$, Sutherland MR, Flores $T J$, Kent AL, Dahlstrom JE, Puelles VG, et al. Development of the human fetal kidney from mid to late gestation in male and female infants. EBioMedicine. 2018;27:275-83.

27. Faa G, Gerosa C, Fanni D, Nemolato S, Locci A, Cabras T, et al. Marked interindividual variability in renal maturation of preterm infants: lessons from autopsy. J Matern Fetal Neonatal Med. 2010;23:129-33.

28. Márquez MG, Cabrera I, Serrano DJ, Sterin-Speziale N. Cell proliferation and morphometric changes in the rat kidney during postnatal development. Anat Embryol. 2002;205:431-40.

29. Bernhardt WM, Schmitt R, Rosenberger C, Münchenhagen PM, Gröne HJ, Frei U, et al. Expression of hypoxia-inducible transcription factors in developing human and rat kidneys. Kidney Int. 2006;69:114-22.

30. Boss JM, Dlouha $H$, Kraus M, Krecek J. The structure of the kidney in relation to age and diet in white rats during the weaning period. J Physiol. 1963;168:196-204. 
31. Velarde V, Humphreys J, Figueroa CD, Vio CP. Postnatal maturation of tissue kallikrein-producing cells (connecting tubule cells) in the rat kidney: a morphometric and immunohistochemical study. Anat Embryol. 1995;192:407-14.

32. Speller AM, Moffat DB. Tubulo-vascular relationships in the developing kidney. J Anat. 1977;123:487-500.

33. Khan S, Cabral PD, Schilling WP, Schmidt ZW, Uddin AN, Gingras A, et al. Kidney proximal tubule lipoapoptosis is regulated by fatty acid transporter-2 (FATP2). J Am Soc Nephrology. 2018;29:81-91.

34. Bedin M, Boyer O, Servais A, Li Y, Villoing-Gaudé L, Tête MJ, et al. Human Cterminal CUBN variants associate with chronic proteinuria and normal renal function. J Clin Investig. 2020;130:335-44.

35. Bachmann S, Metzger R, Bunnemann B. Tamm-Horsfall protein-mRNA synthesis is localized to the thick ascending limb of Henle's loop in rat kidney. Histochemistry. 1990;94:517-23.

36. Castrop $\mathrm{H}$, Schnermann J. Isoforms of renal Na-K-2Cl cotransporter NKCC2: expression and functional significance. Am J Physiol Ren Physiol. 2008;295: F859-66.

37. Syrén ML, Tedeschi S, Cesareo L, Bellantuono R, Colussi G, Procaccio M, et al. Identification of fifteen novel mutations in the SLC12A3 gene encoding the $\mathrm{Na}-\mathrm{Cl}$ Co-transporter in Italian patients with Gitelman syndrome. Hum Mutat. 2002;20:78

38. Sabolić I, Katsura T, Verbavatz JM, Brown D. The AQP2 water channel: effect of vasopressin treatment, microtubule disruption, and distribution in neonatal rats. J Membr Biol. 1995;143:165-75.

39. van Lieburg AF, Knoers NV, Deen PM. Discovery of aquaporins: a breakthrough in research on renal water transport. Pediatr Nephrol. 1995;9:228-34.

40. Ruotsalainen $\mathrm{V}$, Ljungberg $\mathrm{P}$, Wartiovaara J, Lenkkeri $\mathrm{U}$, Kestilä $\mathrm{M}$, Jalanko $\mathrm{H}$, et al. Nephrin is specifically located at the slit diaphragm of glomerular podocytes. Proc Natl Acad Sci USA. 1999;96:7962-7.

41. Boute N, Gribouval O, Roselli S, Benessy F, Lee H, Fuchshuber A, et al. NPHS2, encoding the glomerular protein podocin, is mutated in autosomal recessive steroid-resistant nephrotic syndrome. Nat Genet. 2000;24:349-54.

42. Yamazaki K, Fukata H, Adachi T, Tainaka H, Kohda M, Yamazaki M, et al. Association of increased type I collagen expression and relative stromal overgrowth in mouse epididymis neonatally exposed to diethylstilbestrol. Mol Reprod Dev. 2005;72:291-8.

43. Mourcin F, Breton C, Tellier J, Narang P, Chasson L, Jorquera A, et al. Galectin-1expressing stromal cells constitute a specific niche for pre-Bll cell development in mouse bone marrow. Blood. 2011;117:6552-61.

44. Hata Y, Duh E, Zhang K, Robinson GS, Aiello LP. Transcription factors Sp1 and Sp3 alter vascular endothelial growth factor receptor expression through a novel recognition sequence.J Biol Chem. 1998;273:19294-303.

45. Strickland LA, Jubb AM, Hongo JA, Zhong F, Burwick J, Fu L, et al. Plasmalemmal vesicle-associated protein (PLVAP) is expressed by tumour endothelium and is upregulated by vascular endothelial growth factor-A (VEGF). J Pathol. 2005;206:466-75.

46. Laouedj M, Tardif MR, Gil L, Raquil MA, Lachhab A, Pelletier M, et al. S100A9 induces differentiation of acute myeloid leukemia cells through TLR4. Blood. 2017;129:1980-90.

47. Kaufmann O, Volmerig J, Dietel M. Uroplakin III is a highly specific and moderately sensitive immunohistochemical marker for primary and metastatic urothelial carcinomas. Am J Clin Pathol. 2000;113:683-7.

48. Fujiwara T, Alqadi YW, Okitsu Y, Fukuhara N, Onishi $Y$, Ishizawa $K$, et al. Role of transcriptional corepressor ETO2 in erythroid cells. Exp Hematol. 2013;41:303-15.

49. Raess PW, Paessler ME, Bagg A, Weiss MJ, Choi JK. a-Hemoglobin-stabilizing protein is a sensitive and specific marker of erythroid precursors. Am J Surgical Pathol. 2012;36:1538-47.

50. Lee JW, Chou CL, Knepper MA. Deep sequencing in microdissected renal tubules identifies nephron segment-specific transcriptomes. J Am Soc Nephrology. 2015;26:2669-77.

51. Gabut M, Bourdelais F, Durand S. Ribosome and translational control in stem cells. Cells. 2020;9:497.

52. Liutkute M, Samatova E, Rodnina MV. Cotranslational folding of proteins on the ribosome. Biomolecules. 2020;10:97.

53. Dai X, Zhu M. Coupling of ribosome synthesis and translational capacity with cell growth. Trends Biochem Sci. 2020;45:681-2.

54. Wiggins RC. The spectrum of podocytopathies: a unifying view of glomerular diseases. Kidney Int. 2007:71:1205-14.

55. Shafer MER. Cross-species analysis of single-cell transcriptomic data. Front Cell Dev Biol. 2019;7:175.

56. Jamison RL. Short and long loop nephrons. Kidney Int. 1987;31:597-605.

57. Cha JH, Kim YH, Jung JY, Han KH, Madsen KM, Kim J. Cell proliferation in the loop of henle in the developing rat kidney. J Am Soc Nephrology. 2001;12:1410-21.

58. Chen JK, Nagai K, Chen J, Plieth D, Hino M, Xu J, et al. Phosphatidylinositol 3kinase signaling determines kidney size. J Clin Investig. 2015;125:2429-44.
59. Ren J, Wang $Y$, Liang $Y$, Zhang $Y$, Bao $S, X u$ Z. Methylation of ribosomal protein S10 by protein-arginine methyltransferase 5 regulates ribosome biogenesis. J Biol Chem. 2010;285:12695-705.

60. Ji Z, Kiparaki M, Folgado V, Kumar A, Blanco J, Rimesso G, et al. Drosophila RpS12 controls translation, growth, and cell competition through Xrp1. PLoS Genet. 2019;15:e1008513.

61. Quintero-Ronderos $P$, Laissue $P$. The multisystemic functions of FOXD1 in development and disease. J Mol Med. 2018;96:725-39.

62. Song R, Lopez MLSS, Yosypiv IV. Foxd1 is an upstream regulator of the reninangiotensin system during metanephric kidney development. Pediatr Res. 2017;82:855-62.

63. Sadl V, Jin F, Yu J, Cui S, Holmyard D, Quaggin S, et al. The mouse Kreisler (Krml1/ MafB) segmentation gene is required for differentiation of glomerular visceral epithelial cells. Dev Biol. 2002;249:16-29.

64. Quaggin SE, Vanden Heuvel GB, Igarashi P. Pod-1, a mesoderm-specific basichelix-loop-helix protein expressed in mesenchymal and glomerular epithelial cells in the developing kidney. Mechanisms Dev. 1998;71:37-48.

65. Dong L, Pietsch S, Tan Z, Perner B, Sierig R, Kruspe D, et al. Integration of cistromic and transcriptomic analyses identifies Nphs2, Mafb, and Magi2 as Wilms' tumor 1 target genes in podocyte differentiation and maintenance. J Am Soc Nephrology. 2015;26:2118-28.

66. Terzi F, Burtin M, Hekmati $M$, Jouanneau $C$, Beaufils $H$, Friedlander G. Sodium restriction decreases AP-1 activation after nephron reduction in the rat: role in the progression of renal lesions. Exp Nephrol. 2000;8:104-14.

67. Korsunsky I, Millard N, Fan J, Slowikowski K, Zhang F, Wei K, et al. Fast, sensitive and accurate integration of single-cell data with Harmony. Nat Methods. 2019;16:1289-96

\section{ACKNOWLEDGEMENTS}

We also acknowledge support from Bin Zhao for sequencing and from Aidi Tan and Cong Li for data analyses. In addition, Fangrui Ding thanks Lei Gao and Mo Ding for their care and support.

\section{AUTHOR CONTRIBUTIONS}

$F D, X T$, and JZ designed the study; FD, JM, and BW carried out the experiments; FD, $\mathrm{JM}, \mathrm{XT}$, and $\mathrm{BW}$ analyzed the data; $\mathrm{FD}, \mathrm{XT}$, and $\mathrm{JZ}$ designed the figures; $\mathrm{FD}, \mathrm{XT}, \mathrm{JM}$, $B W$, and JZ drafted and revised the paper; all the authors approved the final version of the manuscript.

\section{FUNDING}

FD is supported by the National Natural Science Foundation of China (No.81800634), and $J Z$ is supported by the Key Technologies R\&D Program of Tianjin (18ZXRHSY0017).

\section{ETHICS STATEMENT}

The animal experiments were approved by the Tianjin Central Hospital of Gynecology and Obstetrics Animal Ethics Committee.

\section{COMPETING INTERESTS}

The authors declare no competing interests.

\section{ADDITIONAL INFORMATION}

Supplementary information The online version contains supplementary material available at https://doi.org/10.1038/s41420-021-00542-9.

Correspondence and requests for materials should be addressed to F.D. or J.Z.

Reprints and permission information is available at http://www.nature.com/ reprints

Publisher's note Springer Nature remains neutral with regard to jurisdictional claims in published maps and institutional affiliations. 
(c) (i) Open Access This article is licensed under a Creative Commons Attribution 4.0 International License, which permits use, sharing, adaptation, distribution and reproduction in any medium or format, as long as you give appropriate credit to the original author(s) and the source, provide a link to the Creative Commons license, and indicate if changes were made. The images or other third party material in this article are included in the article's Creative Commons license, unless indicated otherwise in a credit line to the material. If material is not included in the article's Creative Commons license and your intended use is not permitted by statutory regulation or exceeds the permitted use, you will need to obtain permission directly from the copyright holder. To view a copy of this license, visit http://creativecommons. org/licenses/by/4.0/.

(c) The Author(s) 2021 